\title{
Interactions of a neutrino with an extremely light scalar
}

\author{
Xiao-Gang He a,b, Bruce H.J. McKellar ${ }^{\text {a,1 }}$, G.J. Stephenson Jr. ${ }^{\text {a,c }}$ \\ a School of Physics, University of Melbourne, Parkville, Vic. 3052, Australia \\ ${ }^{\mathrm{b}}$ Department of Physics, National Taiwan University, Taipei 10717, Taiwan \\ ${ }^{\mathrm{c}}$ Department of Physics and Astronomy, University of New Mexico, Albuquerque, NM 87131, USA \\ Received 22 July 1998 \\ Editor: H. Georgi
}

\begin{abstract}
An extremely light scalar weakly interacting with light neutrinos has interesting consequences in stellar evolution, neutrino oscillations and laboratory neutrino mass measurements. In this paper we construct realistic gauge models for such scalar-neutrino interactions. C) 1998 Elsevier Science B.V. All rights reserved.
\end{abstract}

\section{Introduction}

Recently, it has been shown that, if neutrinos weakly interact with an extremely light scalar boson, it is possible to introduce additional MSW like effects in the sun which alter the details of $r$-process nucleosynthesis in supernovae, to impact the earliest formation of stars, and to modify data near the endpoint of the beta spectrum in laboratory measurements of neutrino mass which may aid in the resolution of the "negative mass-squared problem" [1]

The specific renormalizable Lagrangian intro-

\footnotetext{
${ }^{1}$ On leave at Special Research Centre for the Subatomic Structure of Matter, University of Adelaide, SA. 5005, Australia.
}

duced whose phenomenological consequences are discussed in Ref. [1] is of the form

$$
L=\bar{\psi}\left(i \partial-m_{\nu}\right) \psi+\frac{1}{2}\left[\phi\left(\partial^{2}-m_{s}^{2}\right) \phi\right]+g \bar{\psi} \psi \phi
$$

where $\psi$ and $\phi$ are the neutrino and scalar boson, respectively. In order not to be in conflict with other constraints, the scalar mass has to be around [1] $1.3 \times 10^{-18} \mathrm{eV}$, and the coupling $g$ is in the range $3 \times 10^{-21}<|g|<10^{-15}$.

In general the interaction between neutrinos and scalar can also have a renormalizable term of the form

$L=g^{\prime} i \bar{\psi} \gamma_{5} \psi \phi$

This type of interaction, however, will not affect the results obtained in Ref. [1] because, in the macroscopic neutrino background considered there, the 
average of spin of the neutrinos is zero and the interaction term in Eq. (2) will have no effect.

In this paper we study models which extend the standard model by the introduction of a neutrino interaction with an extremely light scalar. In Section 2 , we will discuss some basic features and difficulties of such interactions in gauge models based on the Standard Model (SM). A toy model will be given which also serves to introduce our notation. In Section 3, we will discuss the possibility of realizing such interactions in Majoron models, and in Section 4 , we will discuss the possibilities of realizing such an interaction in models with only Dirac neutrinos.

\section{A simple model}

In the minimal SM it is not possible to accommodate the interactions of Eq. (1) although there is a scalar, the Higgs boson, in the model because this scalar does not couple to neutrinos and, further, experimental data constrain its mass to be larger than $77.5 \mathrm{GeV}$ [2].

In order to get a new scalar interaction like that of Eq. (1), one must go beyond the standard model, while still respecting the $S U(2)_{L} \times U(1)_{Y}$ transformation properties of the existing particles of the standard model. These transformation properties of leptons and the Higgs boson, which are relevant to our discussion, under the SM gauge group $S U(2)_{L} \times$ $U(1)_{Y}$ for electroweak interactions are given by

$L_{L}^{i}=\left(\begin{array}{c}\nu \\ e\end{array}\right)_{L}:\left(2,-\frac{1}{2}\right), \quad E_{R}^{i}=e_{R}^{i}:(1,-1), \quad$ and

$H=\left(\begin{array}{l}h^{0} \\ h^{-}\end{array}\right):\left(2,-\frac{1}{2}\right)$,

where $i=e, \mu, \tau$ is the generation index.

From the quantum numbers of the particles, it is clear that one must introduce new particles to generate the interactions we want. The extremely light scalar which weakly couples to neutrinos will be a new particle beyond the minimal SM. The simplest way to do this is to introduce a real scalar $S$ which transforms under the SM gauge group as a singlet $(1,0)$. This scalar will not couple to the left-handed neutrinos by any renormalizable Lagrangian, so we also introduce right handed neutrinos $\nu_{R}^{i}$ which transform as singlets $(1,0)$, and have a Majorana mass matrix which is introduced as a free parameter. With these new particles, it is possible to have interactions between the new scalar and the neutrinos. Including the Higgs interactions, the relevant terms are

$$
\begin{aligned}
L_{\text {new }}= & -\bar{L}_{L} \frac{m_{\nu}^{\dagger}}{v_{H}} H^{\dagger} \nu_{R}-\bar{\nu}_{R} \frac{m_{\nu}}{v_{H}} H L_{L}-\frac{1}{2} \bar{\nu}_{R}^{c} M^{\dagger} \nu_{R} \\
& -\frac{1}{2} \bar{\nu}_{R} M \nu_{R}^{c}+\bar{\nu}_{R}^{c} C^{\dagger} \nu_{R} S+\bar{\nu}_{R} C \nu_{R}^{c} S \\
& +\frac{1}{2} \partial_{\mu} S \partial^{\mu} S+V(S),
\end{aligned}
$$

where $v_{H}$ is the vacuum expectation value (VEV) of $H$, and $V(S)$ the scalar field potential which has the form

$$
\begin{aligned}
V(S)= & -\frac{1}{2} m_{s}^{2} S^{2}+\text { other terms up to fourth } \\
& \text { power in } S .
\end{aligned}
$$

The generation index on leptons has been suppressed. This Lagrangian of Eqs. (4) and (5) contains some free parameters: a scalar mass $m_{s}$, a scalarneutrino coupling matrix $C$, a Majorana mass matrix $M$ for $\nu_{R}$, and a Dirac mass matrix $m_{\nu}$. Notice that lepton number is explicitly broken in this model. In the neutrino mass eigenstate bases, one then has

$$
\begin{aligned}
L= & \frac{1}{2} \bar{\psi} i \partial \psi-\frac{1}{2} \bar{\psi} \hat{M} \psi+\bar{\psi}_{L} \hat{C} \psi_{R} S+\bar{\psi}_{R} \hat{C}^{\dagger} \psi_{L} S \\
& +\frac{1}{2}\left(\partial_{\mu} S \partial^{\mu} S-m_{s}^{2} s^{2}\right)+\ldots,
\end{aligned}
$$

where $\hat{M}$ is the diagonal mass matrix, $U$ is a unitary matrix which diagonalizes the mass matrix and transforms the coupling matrix $C$ to a new matrix $\hat{C}$ in the following way:

$\hat{M}=U^{T}\left(\begin{array}{ll}0 & m_{\nu}^{T} \\ m_{\nu} & M\end{array}\right) U, \quad \hat{C}=U^{T}\left(\begin{array}{ll}0 & 0 \\ 0 & C\end{array}\right) U$.

The mass eigenstate is $\psi=\psi_{L}+\psi_{R}$, with $\psi_{L}=N_{L}$, $\psi_{R}=N_{L}^{c}$, where

$N_{L}=\left(\begin{array}{l}N_{1 L} \\ N_{2 L}\end{array}\right)=U^{\dagger}\left(\begin{array}{c}\nu_{L} \\ \nu_{R}^{c}\end{array}\right)$,

In this simple model the masses are all free parameters. Using this freedom, it is possible to obtain the parameter ranges used in Ref. [1]. To see how this happens, consider a simple case with just 
one generation. We assume that the light neutrino mass is due to the See-Saw mechanism [3], so that $M \gg m_{\nu}$. The two mass eigenvalues are: $m_{l} \approx$ $-m_{\nu}^{2} / M$ and $m_{h} \approx M$, and $U$ is given by

$U=\left(\begin{array}{cc}\cos \theta & -\sin \theta \\ \sin \theta & \cos \theta\end{array}\right), \quad \sin \theta \approx-\frac{m_{\nu}}{M}$.

The scalar coupling to the light neutrino is given by

$g=\sin ^{2} \theta C \approx\left(\frac{m_{\nu}}{M}\right)^{2} C \approx-\frac{m_{l}}{M} C$.

The upper limit of the electron neutrino mass is constrained to be of order ten $\mathrm{eV} \mathrm{[4]}{ }^{2}$. A value of $g$ in the range of $10^{-21}-10^{-15}$, follows from values of $M$ in the range of $10^{12}-10^{6} \mathrm{GeV}$ if $C$ is of order one.

This exercise is just a demonstration that it is indeed possible to have a model which satisfies the requirements of Ref. [1]. The coupling between the scalar and the light neutrino is naturally small. However, although it is possible to have a small scalar mass due to the arbitrariness of the parameters, it will be much more interesting if some mechanism can be found which guarantees the lightness of the scalar particle.

If the scalar is a Goldstone boson resulting from the breaking of a global symmetry, the scalar is naturally light, $m_{s}=0$. A small mass can develop for the Goldstone boson if the symmetry is explicitly broken weakly due to some mechanism. The Axion [5] is a famous example of this mechanism. However, available Axion models have masses for the spin zero particle which are too large. In the following we consider models in which possible global symmetries are broken spontaneously as well as explicitly due to gravitational effects, such that the would-be Goldstone boson resulting from spontaneous symmetry breaking receives a small mass.

\section{A Majoron model}

In the example given before, the lepton number is explicitly broken by Majorana mass terms and the

\footnotetext{
${ }^{2}$ In the presence of a neutrino cloud, the vacuum neutrino mass can be larger than the experimental bound, which bounds the effective mass inside the cloud [1].
}

scalar coupling terms. We now consider a model in which lepton number is spontaneously broken with a massless Majoron. The model to be studied contains in addition to the usual minimal SM particle contents, right-handed singlet neutrinos $\nu_{R}^{i}$, one triplet Higgs $\chi$ and a singlet scalar $S$. The transformation properties under the $S U(2)_{L} \times U(1)_{Y} \times U(1)_{\text {lepton }}$ group for scalar particles are:

$H:(2,-1 / 2)(0) ; \quad \chi:(3,1)(-2)$;

$S:(1,0)(-2)$.

Here the quantum number in the last bracket is the lepton number. Lepton number is spontaneously broken when $\chi$ and $S$ develop non-zero vacuum expectation values $v_{\chi}$ and $v_{S}$, respectively. The Majoron field is given by

$$
\begin{aligned}
\phi= & N\left[2 v_{\chi}^{2} v_{H} \mathfrak{\Im}\left(h^{0}\right)-v_{H}^{2} v_{\chi} \mathfrak{\Im}(\chi)\right. \\
& \left.-\left(v_{H}^{2}+4 v_{\chi}^{2}\right) v_{S} \mathfrak{\Im} S\right],
\end{aligned}
$$

where

$N=1 / \sqrt{\left(2 v_{\chi}^{2} v_{H}\right)^{2}+\left(v_{H}^{2} v_{\chi}\right)^{2}+\left(\left(v_{H}^{2}+4 v_{\chi}^{2}\right) v_{S}\right)^{2}}$

is the normalization constant.

The mass matrix for the neutrinos in the bases $\left(\nu_{L}, \nu_{R}^{c}\right)$ is given by

$M_{\text {neutrino }}=\left(\begin{array}{ll}m & m_{D}^{T} \\ m_{D} & M\end{array}\right)$,

where $m \sim v_{\chi}, m_{D} \sim v_{H}$ and $M \sim v_{S}$ are the Majorana mass matrix for the left-handed neutrinos, the Dirac mass matrix for neutrinos and the Majorana mass matrix for the right-handed neutrinos, respectively.

The Majoron-neutrino coupling matrix is given by

$C=\frac{N_{\psi}}{\sqrt{2}}\left(\begin{array}{cc}-m v_{H}^{2} & 2 m_{D}^{T} v_{\chi}^{2} \\ 2 m_{D} v_{\chi}^{2} & -M\left(v_{H}^{2}+4 v_{\chi}^{2}\right)\end{array}\right)$

For simplicity we work with just one generation and assume $m, m_{D}$ are real and $M$ has a phase of ${ }^{3}$ $\pi / 2$. It has long been known that a Majoron model

\footnotetext{
${ }^{3}$ Without complex phases, the Majoron being a pseudoscalar couples to neutrinos through the $\gamma_{5}$ coupling of Eq. (2). Complex phases in the interaction are needed to induce the scalar coupling of Eq. (1). We choose a phase of $\pi / 2$ to maximize the effect.
} 
of the type discussed here without the singlet is ruled out by experimental data from LEP on $\mathrm{Z}$ decay width [4], because of additional light charged scalar decay modes for Z [6]. This problem can be solved by the introduction of the singlet scalar, with $v_{S} \gg$ $v_{H} \gg v_{\chi}$ such that the light charged scalar decay modes of $\mathrm{Z}$ are suppressed. We will assume this is the case in the discussion to follow.

After diagonalization of the neutrino mass matrix and in the mass eigenstate bases, the light neutrino mass and interaction with the scalar is given by

$$
\begin{aligned}
L= & -\frac{1}{2} \bar{\psi} m_{l} \psi-\frac{1}{2 \sqrt{2} v_{S}} \\
& \times\left[i \bar{\psi}\left(m_{1}+\frac{2 m_{D}^{2}}{M} \sin \delta_{1}\right) \gamma_{5} \psi\right. \\
& \left.-\frac{2 m_{D}^{2}}{M} \cos \delta_{1} \bar{\psi} \psi\right] \phi,
\end{aligned}
$$

where $m_{1} \approx \sqrt{m^{2}+\left(m_{D}^{2} / M\right)^{2}}$ is the eigenmass and $\delta_{1}=\operatorname{arctg}\left(m_{D}^{2} / m M\right)$.

As has been discussed before, the interaction term with $\gamma_{5}$ does not affect the results of interest to us, so we can ignore it in the remainder of this section. We then identify

$g=\frac{m_{D}^{2}}{\sqrt{2} M v_{S}} \cos \delta_{1}$.

It is easy to obtain a neutrino mass close to the upper limit of order ten $\mathrm{eV}$. Also if we assume $m$ is the same order of magnitude as $m_{D}^{2} / M, \cos \delta_{1}$ is of order one, $M \sim v_{S}$ is in the range of $10^{6}$ to $10^{12}$ $\mathrm{GeV}$, and the coupling $g$ is in the range of $10^{-15}$ $10^{-21}$.

So far the Majoron mass is exactly zero. However, this may not hold when gravitational effects are considered. It has been argued that, in the presence of gravity, the lepton number symmetry may be explicitly broken and result in a small mass for Majoron [7]. The mass would be inversely proportional to the Plank mass and therefore can be made very small. The lowest terms have dimension five. To this order, a number of terms can contribute. For example a term of the following form can appear in the potential

$$
V_{\text {add }}=\beta \frac{1}{m_{\mathrm{Pl}}}\left(\chi^{+} \chi\right)^{2} S+\text { H.C. },
$$

where $m_{\mathrm{Pl}}$ is the Planck mass. This term satisfies the condition that when the Planck mass goes to infinity, its contribution vanishes. Due to the appearance of this term, the minimal conditions for the potential change, and the Majoron $\phi$ will mix with the massive scalar and also become massive. The masssquared $m_{\phi}^{2}$ is given by

$m_{\phi}^{2}=-\beta \frac{v_{\chi}^{4}}{2 \sqrt{2} v_{s} m_{\mathrm{Pl}}}$.

This mass can be easily made to be around $10^{-18}$ $\mathrm{eV}$ with $\beta$ to be of order one. The analysis with additional terms can be easily carried out in the same way.

\section{A model for Dirac neutrino - scalar interac- tions}

The conditions required for neutrino-scalar coupling of the type of Eq. (1) can also be realized for Dirac neutrinos. In the following we present a simple model to demonstrate this fact. In this model we introduce a new global $U(1)$ symmetry whose quantum numbers for different fields are indicated in the second bracket in the following:

$$
\begin{array}{lll}
l_{L}^{i} & : & (2,-1 / 2)(0) ; \quad \nu_{R}^{i}:(1,0)\left(\alpha_{i}\right), \\
H_{j} & : & \left(2,-\frac{1}{2}\right)\left(\beta_{j}\right) ; \quad S:(1,0)(1) ; \\
\alpha_{1}= & 0 ; \quad \alpha_{2}=\alpha_{3}=1 ; \quad \beta_{1}=0 ; \quad \beta_{2}=1 .
\end{array}
$$

Here $i$ is the lepton generation index and $j$ is doublet Higgs index, which is introduced because we find that two Higgs doublets are needed. The global symmetry is broken by VEV's of $H_{j}$ and $S$. The corresponding Goldstone boson is given by

$$
\begin{aligned}
\phi= & N\left[v_{H_{2}}^{2} v_{H_{1}} \mathfrak{I}\left(h_{1}^{0}\right)-v_{H_{1}}^{2} v_{H_{2}} \mathfrak{I}\left(h_{2}^{0}\right)\right. \\
& \left.-\left(v_{H_{1}}^{2}+v_{H_{2}}^{2}\right) v_{S} \mathfrak{I}(S)\right],
\end{aligned}
$$


where

$N=1 / \sqrt{\left(v_{H_{2}}^{2} v_{H_{1}}\right)^{2}+\left(v_{H_{1}}^{2} v_{H_{2}}\right)^{2}+\left(\left(v_{H_{1}}^{2}+v_{H_{2}}^{2}\right) v_{S}\right)^{2}}$ is the normalization constant.

The mass matrix and $\phi$ neutrino coupling matrix are given by

$M=\left(\begin{array}{lll}m_{11} & m_{12} & m_{13} \\ m_{21} & m_{22} & m_{23} \\ m_{31} & m_{32} & m_{33}\end{array}\right)$,

$C=N\left(\begin{array}{ccc}m_{11} v_{H_{2}}^{2} & -m_{12} v_{H_{1}}^{2} & -m_{13} v_{H_{1}}^{2} \\ m_{21} v_{H_{2}}^{2} & m_{22} v_{H_{2}}^{2} & -m_{23} v_{H_{1}}^{2} \\ m_{31} v_{H_{2}}^{2} & -m_{32} v_{H_{1}}^{2} & m_{33} v_{H_{2}}^{2}\end{array}\right)$

The mass matrix can be diagonalized by bi-unitary diagonalization, that is

$\hat{m}=\left(\begin{array}{ccc}m_{1} & 0 & 0 \\ 0 & m_{2} & 0 \\ 0 & 0 & m_{3}\end{array}\right)=V_{L} M V_{R}$,

which gives a transformed, coupling matrix

$\hat{C}=V_{L} C V_{R}$.

To illustrate this model, consider an example with two generations. The coupling matrix is of the form

$$
\begin{aligned}
& \hat{C}=-N m_{12}\left(v_{H_{1}}^{2}+v_{H_{2}}^{2}\right) e^{i \delta_{R}}\left(\begin{array}{ll}
c_{L} s_{R} & c_{L} c_{R} \\
s_{L} s_{R} & s_{L} c_{R}
\end{array}\right) \\
& V_{L}=\left(\begin{array}{cc}
c_{L} & -s_{L} e^{i \delta_{L}} \\
s_{L} & c_{L} e^{i \delta_{L}}
\end{array}\right), \quad V_{R}=\left(\begin{array}{cc}
c_{R} & -s_{R} \\
s_{R} e^{i \delta_{R}} & c_{R} e^{i \delta_{R}}
\end{array}\right)
\end{aligned}
$$

where $c_{L, R}=\cos \theta_{L, R}$ and $s_{L, R}=\sin \theta_{L, R}$. This model is very different from the Majoron model discussed previously. At least two generations with mixing are needed. The Goldstone boson coupling to the lightest neutrino is given by

$g=\frac{m_{12}}{v_{S}} \sin \delta_{R} c_{L} s_{R}$.

With $m_{12}$ in the eV range, $c_{L} s_{R}$ of order one and $v_{S}$ in the range $10^{6}-10^{12} \mathrm{GeV}$, it is easy to obtain $g$ in the desired range of $10^{-15}-10^{-21}$.
Again the Goldstone boson mass becomes nonzero when gravitational effects are considered. As an example we introduce a term of the form

$V_{\mathrm{add}}=\beta \frac{\left(H_{2}^{\dagger} H_{2}\right)^{2} S}{m_{\mathrm{Pl}}}+$ H.C.

in the potential. The field $\phi$ develops a mass given by

$m_{\phi}^{2}=\beta \frac{v_{H_{2}}^{4}}{v_{s} m_{\mathrm{Pl}}}$,

which can easily be in the desired range.

\section{Conclusion}

Neutrinos that interact weakly with an extremely light scalar have many interesting consequences in stellar evolution, neutrino oscillations and laboratory neutrino mass measurements. In this paper we have study the possibility of realizing such interactions in gauge models. We have shown that it is indeed possible to construct such models, and that it is possible to arrange for the new scalar to be very light and for its coupling to neutrinos to be naturally very small. Models which exhibit this behaviour are constructed in which the neutrinos are either Majorana or Dirac particles.

\section{Acknowledgements}

This work was supported in part by the Australian Research Council and by National Science Council of ROC (NSC 87-2811-M-002-046). B.H.J.M. thanks the Department of Physics of the National Taiwan University, and the National Center for Theoretical Sciences, Taiwan for hospitality where part of this work was done. G.J.S. thanks the Division of International Programs of the National Science Foundation for travel support and the University of Melbourne for its hospitality. We thank Ray Volkas for helpful discussions. 


\section{References}

[1] G.J. Stephenson Jr., T. Goldman, B.H.J. McKellar, Intl. J. of Mod. Phys. A 13 (1998) 2765, hep-ph/9603392; Mod. Phys. Lett. A 12 (1997) 2391, hep-ph/9610317.

[2] P. Bock et al., ALEPH, DELPHI, L3 and OPAL Collaborations, CERN/ep-98-046.

[3] M. Gell-Mann, P. Ramond, R. Slansky, in: D. Freedman et al. (Eds.), Supergravity, North-Holland, Amsterdam, 1979; T. Yanagida, KEK Lectures, 1979.
[4] Particle Data Group, Phys. Rev. D 54 (1996) 1.

[5] R. Peccei, H. Quinn, Phys. Rev. Lett. 38 (1977) 1440; Phys. Rev. D 16 (1977) 1791; H.Y. Cheng, Phys. Rep. 159 (1988) 1.

[6] G. Gelmini, M. Roncadelli, Phys. Lett. B 99 (1981) 411; H. Georgi, S. Glashow, S. Nussinov, Nucl. Phys. B 193 (1981) 297.

[7] E. Akhmedov, Z. Berezhiani, R. Mohapatra, G. Senjanovic, Phys. Lett. B 299 (1993) 90; I. Rothestein, K.S. Babu, D. Seckel, Phys. Lett. B 300 (1993) 367. 